Proceedings of the 2012 Winter Simulation Conference

C. Laroque, J. Himmelspach, R. Pasupathy, O. Rose, and A. M. Uhrmacher, eds.

\title{
TIGHT BOUNDS FOR AMERICAN OPTIONS VIA MULTILEVEL MONTE CARLO
}

\author{
Denis Belomestny \\ Duisburg-Essen University \\ Forsthausweg 2 \\ D-47057 Duisburg, GERMANY
}

\author{
Marcel Ladkau \\ Weierstrass Institute Berlin \\ Mohrenstrasse 39 \\ D-10117 Berlin, GERMANY
}

\author{
John Schoenmakers \\ Weierstrass Institute Berlin \\ Mohrenstrasse 39 \\ D-10117 Berlin, GERMANY
}

\begin{abstract}
This paper is an overview of recent results by Belomestny and Schoenmakers 2011 and Belomestny, Ladkau, and Schoenmakers 2012, on dual and primal Monte Carlo evaluation of American style derivatives using multilevel principles. It presents a novel and generic approach to reduce the complexity of nested simulations problems arising in Monte Carlo pricing of American options. The approach genuinely uses the multilevel idea where each level corresponds to a given number of inner simulations. A thorough complexity analysis of the respective nested dual algorithm and nested policy improvement algorithm shows that a significant complexity reduction can be achieved by using the multilevel versions of the algorithms.
\end{abstract}

\section{INTRODUCTION}

Pricing high-dimensional American options in an efficient way has been a challenge for decades. For low or moderate dimensions, deterministic (PDE) based methods may be applicable, but for higher dimensions Monte Carlo based methods are practically the only way out. Besides the dimension independent convergence rates, Monte Carlo methods are also popular because of their generic applicability. In the late nineties several regression methods for constructing "good" exercise policies yielding price lower bounds were introduced in the literature (see Carriere 1996, Longstaff and Schwartz 2001, and Tsitsiklis and Van Roy 2001, for a detailed description see also Glasserman 2004). Among many other approaches we mention that Broadie and Glasserman 2004 developed a stochastic mesh method, Bally and Pages 2003 introduced quantization methods, and Kolodko and Schoenmakers 2006 considered a class of policy iterations. In Bender, Kolodko, and Schoenmakers 2008 it is demonstrated that the latter approach can be combined effectively with the Longstaff-Schwartz approach.

The methods mentioned above commonly provide a (generally suboptimal) exercise policy, hence a lower price for an American product. They are therefore called primal methods. As a next breakthrough in the Monte Carlo simulation of American options, a dual approach was developed by Rogers 2002 and independently by Haugh and Kogan 2004, related to earlier ideas in Davis and Karatzas 1994. Due to the dual formulation one considers "good" martingales rather than "good" stopping times. In fact, based on a "good" martingale the price of an American derivative can be bounded from above by a "look-back" option due to the difference of the cash-flow and this martingale. Probably one of the most popular numerical methods for computing dual upper bounds is the method of Andersen and Broadie 2004. However, this method has a drawback, namely a high computational complexity due to the need for nested Monte Carlo 


\section{Belomestny, Ladkau, and Schoenmakers}

simulations. As a remedy, Belomestny, Bender, and Schoenmakers 2009 developed a non-nested simulation algorithm using regression for approximating the integrand of a suitable martingale representation.

In this paper we review two new multilevel Monte Carlo simulation approaches where the multilevel concept is applied to the number of inner Monte Carlo simulations, rather than the discretization step size as in Giles 2008. While the first approach relies on the dual method leading to a multilevel version of the Andersen and Broadie 2004 algorithm, the second one leads to a multilevel version of the policy iteration approach presented in Kolodko and Schoenmakers 2006. Regarding the latter part only standard (Howard) policy iteration is considered, but, with no doubt the method may be applied successfully to the more refined policy iteration procedure in Kolodko and Schoenmakers 2006 as well. Finally we note that in this review all theorems are stated without proofs. For proofs and further details we refer to Belomestny and Schoenmakers 2011 and Belomestny, Ladkau, and Schoenmakers 2012, respectively.

\section{PRIMAL AND DUAL VALUATION OF AMERICAN OPTIONS}

Let $\left(Z_{j}\right)_{j \geq 0}$ be a nonnegative adapted process on a filtered probability space $\left(\Omega, \mathbb{F}=\left(\mathscr{F}_{j}\right)_{j \geq 0}, \mathbb{P}\right)$ representing the discounted payoff of an American option, so that the holder of the option receives $Z_{j}$ if the option is exercised at time $j \in\{0, \ldots, T\}$ with $T \in \mathbb{N}_{+}$. The pricing of American options can be formulated as a primal-dual problem. Let $Y_{j}$ denote the time $j$ solution to this problem. The primal representation corresponds to the following optimal stopping problems

$$
Y_{j}^{*}:=\max _{\tau \in \mathscr{T}[j, \ldots, T]} \mathbb{E}_{\mathscr{F}_{j}}\left[Z_{\tau}\right], \quad j=0, \ldots, T,
$$

where $\mathscr{T}[j, \ldots, T]$ is the set of $\mathbb{F}$-stopping times taking values in $\{j, \ldots, T\}$. The process $\left(Y_{j}^{*}\right)_{j \geq 0}$ is called the Snell envelope. It is well known that $Y^{*}$ is a supermartingale satisfying the Bellman principle

$$
Y_{j}^{*}=\max \left(Z_{j}, \mathbb{E}_{\mathscr{F}_{j}}\left[Y_{j+1}^{*}\right]\right), \quad 0 \leq j<T, \quad Y_{T}^{*}=Z_{T} .
$$

An exercise policy is a family of stopping times $\left(\tau_{j}\right)_{j=0, \ldots, T}$ such that $\tau_{j} \in \mathscr{T}[j, \ldots, T]$.

During the nineties the primal approach was the only method available. Some years later a quite different "dual" approach was discovered by Rogers 2002 and Haugh and Kogan 2004. The next theorem summarizes their results.

Theorem 1 Let $\mathscr{M}$ denote the space of adapted martingales, then we have the following dual representation for the value process $Y_{j}^{*}$

$$
\begin{aligned}
Y_{j}^{*} & =\inf _{\pi \in \mathscr{M}} \mathbb{E}_{\mathscr{F}_{j}}\left[\max _{s \in\{j, \ldots, T\}}\left(Z_{s}-\pi_{s}+\pi_{j}\right)\right] \\
& =\max _{s \in\{j, \ldots, T\}}\left(Z_{s}-\pi_{s}^{*}+\pi_{j}^{*}\right) \quad \text { a.s., }
\end{aligned}
$$

where

$$
Y_{j}^{*}=Y_{0}^{*}+\pi_{j}^{*}-A_{j}^{*}
$$

is the (unique) Doob decomposition of the supermartingale $Y_{j}^{*}$. That is, $\pi^{*}$ is a martingale and $A^{*}$ is an increasing process with $\pi_{0}=A_{0}=0$ such that (1) holds.

\section{UPPER AND LOWER BOUNDS FOR BERMUDAN OPTIONS VIA NESTED MONTE CARLO}

Assume that the cash-flow $Z_{j}$ is of the form (with a slight abuse of notation) $Z_{j}=Z_{j}\left(X_{j}\right)$ for some underlying (possibly high-dimensional) Markovian process $X$. As a consequence, the Snell envelope then has the form $Y_{j}^{*}=Y_{j}^{*}\left(X_{j}\right), j=0, \ldots, T$, as well. Furthermore, it is assumed that we are given a stopping family $\left(\tau_{j}\right)$ that is consistent, i.e.

$$
\tau_{j}>j \Rightarrow \tau_{j}=\tau_{j+1}, \quad j=0, \ldots, T-1
$$




\section{Belomestny, Ladkau, and Schoenmakers}

and that $\left(\tau_{j}\right)$ depends on $\omega$ only through the path $X$. in the following way. For each $j$ the event $\left\{\tau_{j}=j\right\}$ is measurable w.r.t. $\sigma\left\{X_{j}\right\}$, and $\tau_{j}$ is measurable w.r.t. $\sigma\left\{X_{k}, j \leq k \leq T\right\}$, i.e.,

$$
\tau_{j}(\omega)=h_{j}\left(X_{j}(\omega), \ldots, X_{T}(\omega)\right)
$$

for some Borel measurable function $h_{j}$. A typical example of such a stopping family is

$$
\tau_{j}=\inf \left\{k: j \leq k \leq T, Z_{k}\left(X_{k}\right)>f_{k}\left(X_{k}\right)\right\} \wedge T,
$$

for a set of real valued functions $f_{k}(x)$, with $j=0, \ldots, T$, and $\wedge$ denoting the minimum operator. The stopping policy defines a lower bound for $Y^{*}$ via

$$
Y_{j}=\mathbb{E}_{\mathscr{F}_{j}}\left[Z_{\tau_{j}}\right], \quad j=0, \ldots, T
$$

Consider now a new family $\left(\widehat{\tau}_{j}\right)_{j=0, \ldots, T}$ defined by

$$
\widehat{\tau}_{j}:=\inf \left\{k: j \leq k<T, Z_{k} \geq \mathbb{E}_{\mathscr{F}_{k}}\left[Z_{\tau_{k+1}}\right]\right\} \wedge T .
$$

The basic idea behind (3) goes back to Howard 1960 in fact. For more general versions of policy iteration and their analysis, see Kolodko and Schoenmakers 2006. Next, we introduce the $\left(\mathscr{F}_{j}\right)$-martingale

$$
\pi_{j}=\sum_{k=1}^{j}\left(\mathbb{E}_{\mathscr{F}_{k}}\left[Z_{\tau_{k}}\right]-\mathbb{E}_{\mathscr{F}_{k-1}}\left[Z_{\tau_{k}}\right]\right), \quad j=0, \ldots, T,
$$

and then consider,

$$
\widetilde{Y}_{j}:=\mathbb{E}_{\mathscr{F}_{j}}\left[\max _{k=j, \ldots, T}\left(Z_{k}-\pi_{k}+\pi_{j}\right)\right]
$$

along with

$$
\widehat{Y}_{j}:=\mathbb{E}_{\mathscr{F}_{j}}\left[Z_{\widehat{\tau}_{j}}\right], \quad j=0, \ldots, T .
$$

The following theorem states that $\widehat{Y}$ is an improvement of $Y$ and that the Snell envelope process $Y_{j}^{*}$ lies between $\widehat{Y}_{j}$ and $\widetilde{Y}_{j}$ with probability 1 .

Theorem 2 It holds

$$
Y_{j} \leq \widehat{Y}_{j} \leq Y_{j}^{*} \leq \widetilde{Y}_{j}, \quad j=0, \ldots, T \quad \text { a.s. }
$$

The main issue in the Monte Carlo construction of $\widehat{Y}$ and $\widetilde{Y}$ is the estimation of the conditional expectations in (3) and (4). A canonical approach is the use of sub-simulations. In this respect we consider an enlarged probability space $\left(\Omega, \mathbb{F}^{\prime}, \mathbb{P}\right)$, where $\mathbb{F}^{\prime}=\left(\mathscr{F}_{j}^{\prime}\right)_{j=0, \ldots, T}$ and $\mathscr{F}_{j} \subset \mathscr{F}_{j}^{\prime}$ for each $j$. By assumption, $\mathscr{F}_{j}^{\prime}$ specified as

$$
\mathscr{F}_{j}^{\prime}=\mathscr{F}_{j} \vee \sigma\left\{X^{i, X_{i}}, i \leq j\right\} \text { with } \mathscr{F}_{j}=\sigma\left\{X_{i}, i \leq j\right\},
$$

where for a generic $\left(\omega, \omega_{i n}\right) \in \Omega, X^{i, X_{i}}:=X_{k}^{i, X_{i}(\omega)}\left(\omega_{i n}\right), k \geq i$ denotes a sub trajectory starting at time $i$ in the state $X_{i}(\omega)=X_{i}^{i, X_{i}(\omega)}$ of the outer trajectory $X(\omega)$. In particular, the random variables $X^{i, X_{i}}$ and $X^{i^{\prime}, X_{i^{\prime}}}$ are by assumption independent, conditionally $\left\{X_{i}, X_{i^{\prime}}\right\}$, for $i \neq i^{\prime}$. On the enlarged space we consider $\mathscr{F}_{j}^{\prime}$ measurable estimations $\mathscr{C}_{j, M}$ of $C_{j}=\mathbb{E}_{\mathscr{F}_{j}}\left[Z_{\tau_{j+1}}\right]$ as being standard Monte Carlo estimates based on $M$ sub simulations. More precisely

$$
\mathscr{C}_{j, M}=\frac{1}{M} \sum_{m=1}^{M} Z_{\tau_{j+1}^{(m)}}\left(X_{\tau_{j+1}^{j, X_{j}}}^{(m)}\right)
$$




\section{Belomestny, Ladkau, and Schoenmakers}

where

$$
\tau_{j+1}^{(m)}=h_{j+1}\left(X_{j+1}^{j, X_{j},(m)}, \ldots, X_{T}^{j, X_{j},(m)}\right), \quad 0 \leq j<T
$$

are evaluated on $M$ sub trajectories all starting at time $j$ in $X_{j}$. Obviously, $\mathscr{C}_{j, M}$ is an unbiased estimator for $C_{j}$ with respect to $\mathbb{E}_{\mathscr{F}_{j}}[\cdot]$. We thus end up with simulation based versions of (3) and (4) respectively,

$$
\begin{aligned}
\widehat{\tau}_{j, M}:=\inf \{k: j \leq & \left.k<T, Z_{k}>\mathscr{C}_{k, M}\right\} \wedge T, \quad j=0, \ldots, T, \\
\pi_{j, M}:= & \sum_{k=1}^{j}\left(Z_{k}-\mathscr{C}_{k-1, M}\right) 1_{\left\{\tau_{k}=k\right\}} \\
& +\sum_{k=1}^{j}\left(\mathscr{C}_{k, M}-\mathscr{C}_{k-1, M}\right) 1_{\left\{\tau_{k}>k\right\}} .
\end{aligned}
$$

Denote

$$
\widehat{Y}_{j, M}:=\mathbb{E}_{\mathscr{F}_{j}}\left[Z_{\widehat{\tau}_{j, M}}\right], \quad j=0, \ldots, T
$$

and

$$
\widetilde{Y}_{j, M}:=\mathbb{E}_{\mathscr{F}_{j}}\left[\max _{k=j, \ldots, T}\left(Z_{k}-\pi_{k, M}+\pi_{j, M}\right)\right] .
$$

Concerning the properties of $\widehat{Y}_{j, M}$ and $\widetilde{Y}_{j, M}$ one can prove the following results under mild regularity conditions.

Theorem 3 Let us assume that there exist constants $B_{0}>0, B_{1}>0$ and $\alpha>0$, such that for any $\delta>0$ and $j=0, \ldots, T-1$,

$$
\mathbb{P}\left(\left|\mathbb{E}_{\mathscr{F}_{j}}\left[Z_{\tau_{j+1}}\right]-Z_{j}\right| \leq \delta\right) \leq B_{0} \delta^{\alpha}, \quad\left|Z_{j}\right|<B_{1}
$$

It then holds,

$$
\left|\widehat{Y}_{0}-\widehat{Y}_{0, M}\right| \leq M^{-\frac{1+\alpha}{2}} B
$$

with some constant $B$ depending only on $\alpha, B_{0}$ and $B_{1}$. Moreover

$$
\mathbb{E}\left[\left(Z_{\widehat{\tau}_{0, M}}-Z_{\widehat{\tau}_{0}}\right)^{2}\right] \leq C M^{-\alpha / 2} .
$$

for some $C>0$.

Theorem 4 Introduce for $\mathscr{Z}:=\max _{j=0, \ldots, T}\left(Z_{j}-\pi_{j}\right)$, the random set

$$
\mathscr{Q}=\left\{j: Z_{j}-\pi_{j}=\mathscr{Z}\right\},
$$

and the $\mathscr{F}_{T}$ measurable random variable

$$
\Lambda:=\min _{j \notin \mathscr{Q}}\left(\mathscr{Z}-Z_{j}+\pi_{j}\right),
$$

with $\Lambda:=+\infty$ if $\mathscr{Q}=\{0, \ldots, T\}$. Obviously $\Lambda>0$ a.s. Further suppose that

$$
\mathbb{E}\left[\Lambda^{-\xi}\right]<\infty \text { for some } 0<\xi \leq 1, \quad \text { and } \# \mathscr{Q}=1 .
$$

It then holds,

$$
\left|\widetilde{Y}_{0}-\widetilde{Y}_{0, M}\right| \leq C M^{-\frac{\xi+1}{2}}
$$

for some constant $C$.

Example 1 Let us assume that $\Lambda$ has a density $g$ that is continuous and finite in a right neighborhood of zero. We then have

$$
\mathbb{E} \frac{1}{\Lambda^{\xi}}=\int_{0}^{\infty} z^{-\xi} g(z) d z<\infty \text { for any } 0 \leq \xi<1 .
$$




\section{Belomestny, Ladkau, and Schoenmakers}

\section{POLICY IMPROVED LOWER BOUND BY MULTILEVEL MONTE CARLO}

For a fixed natural number $L$ and a set of natural numbers $\mathbf{m}:=\left(m_{0}, \ldots, m_{L}\right)$ satisfying $1 \leq m_{0}<\ldots<m_{L}$, we consider in the spirit of Giles 2008 the telescoping sum

$$
\widehat{Y}_{m_{L}}=\widehat{Y}_{m_{0}}+\sum_{l=1}^{L}\left(\widehat{Y}_{m_{l}}-\widehat{Y}_{m_{l-1}}\right)
$$

where $\widehat{Y}_{m}:=\widehat{Y}_{0, m}$. Next we take a set of natural numbers $\mathbf{n}:=\left(n_{0}, \ldots, n_{L}\right)$ satisfying $n_{0}>\ldots>n_{L} \geq 1$, and simulate an initial set of cash-flows

$$
\left\{Z_{\widetilde{\tau}_{m_{0}}}^{(j)}, \quad j=1, \ldots, n_{0}\right\},
$$

due to an initial set of trajectories $\left\{X^{0, x,(j)}, j=1, \ldots, n_{0}\right\}$, where

$$
Z_{\widehat{\tau}_{m_{0}}}^{(j)}:=Z_{\widehat{\tau}_{0, m_{0}}^{(j)}}\left(X_{\widehat{\tau}_{0, m_{0}}}^{0, x,(j)}\right)
$$

Next we simulate independently for each level $l=1, \ldots, L$, a set of pairs

$$
\left\{\left(Z_{\widehat{\tau}_{m_{l}}}^{(j)}, Z_{\widehat{\tau}_{m_{l-1}}}^{(j)}\right), \quad j=1, \ldots, n_{l}\right\}
$$

due to a set of trajectories $X^{0, x,(j)}, j=1, \ldots, n_{l}$, to obtain the multilevel estimator

$$
\widehat{\mathscr{Y}}_{\mathbf{n}, \mathbf{m}}:=\frac{1}{n_{0}} \sum_{j=1}^{n_{0}} Z_{\widehat{\tau}_{m_{0}}}^{(j)}+\sum_{l=1}^{L} \frac{1}{n_{l}} \sum_{j=1}^{n_{l}}\left(Z_{\widehat{\tau}_{m_{l}}}^{(j)}-Z_{\widehat{\tau}_{m_{l-1}}}^{(j)}\right)
$$

as an approximation to $\widehat{Y}$. Henceforth we always take $\mathbf{m}$ to be a geometric sequence $m_{l}=m_{0} \kappa^{l}$, for some $m_{0}, \kappa \in \mathbb{N}, \kappa \geq 2$.

\section{DUAL UPPER BOUND BY MULTILEVEL MONTE CARLO}

With the notations of the previous section we define

$$
\widetilde{Y}_{m_{L}}=\widetilde{Y}_{m_{0}}+\sum_{l=1}^{L}\left[\widetilde{Y}_{m_{l}}-\widetilde{Y}_{m_{l-1}}\right]
$$

where $\widetilde{Y}_{m}:=\widetilde{Y}_{0, m}$. Given a sequence $\mathbf{n}=\left(n_{0}, \ldots, n_{L}\right)$ with $n_{0}>\ldots>n_{L} \geq 1$, we then simulate for $l=0$ an initial set of trajectories

$$
\left\{\left(Z_{j}^{(i)}, \pi_{j, m_{0}}^{(i)}\right), \quad i=1, \ldots, n_{0}, \quad j=0, \ldots, T,\right\}
$$

of the two-dimensional vector process $\left(Z_{j}, \pi_{j, m_{0}}\right)$, and then for each level $l=1, \ldots, L$, independently, a set of trajectories

$$
\left\{\left(Z_{j}^{(i)}, \pi_{j, m_{l-1}}^{(i)}, \pi_{j, m_{l}}^{(i)}\right), \quad i=1, \ldots, n_{l}, \quad j=0, \ldots, T\right\}
$$

of the vector process $\left(Z_{j}, \pi_{j, m_{l-1}}, \pi_{j, m_{l}}\right)$. Based on this simulation we consider the following multilevel estimator:

$$
\widetilde{\mathscr{Y}}_{\mathbf{n}, \mathbf{m}}:=\frac{1}{n_{0}} \sum_{i=1}^{n_{0}} \mathscr{Z}_{m_{0}}^{(i)}+\sum_{l=1}^{L} \frac{1}{n_{l}} \sum_{i=1}^{n_{l}}\left[\mathscr{Z}_{m_{l}}^{(i)}-\mathscr{Z}_{m_{l-1}}^{(i)}\right]
$$

with $\mathscr{Z}_{m_{l}}^{(i)}:=\max _{j=0, \ldots, T}\left(Z_{j}^{(i)}-\pi_{j, m_{l}}^{(i)}\right), i=1, \ldots, n_{l}, l=0, \ldots, L$. 


\section{Belomestny, Ladkau, and Schoenmakers}

\section{Complexity analysis}

Let us now compute the numerical complexity of the multilevel estimators (5) and (6). To this end we consider a "generic" multi-level estimator for a target quantity $X$ of the form:

$$
X_{\mathbf{n}, \mathbf{m}}:=\frac{1}{n_{0}} \sum_{i=1}^{n_{0}} \mathscr{X}_{m_{0}}^{(i)}+\sum_{l=1}^{L} \frac{1}{n_{l}} \sum_{i=1}^{n_{l}}\left[\mathscr{X}_{m_{l}}^{(i)}-\mathscr{X}_{m_{l-1}}^{(i)}\right]
$$

where for any fixed $l$ the random variables $\mathscr{X}_{m_{l}}^{(i)}, i=1, \ldots, n_{l}$, are i.i.d. and the pairs $\left(\mathscr{X}_{m_{l}}^{(i)}, \mathscr{X}_{m_{l-1}}^{(i)}\right)$, $l=1, \ldots, L$, are independent. Assume that there are some positive constants $\gamma, \beta, \mu_{\infty}, \sigma_{\infty}$ and $\mathscr{V}_{\infty}$ such that $\operatorname{Var}\left[\mathscr{X}_{m}\right] \leq \sigma_{\infty}^{2}$

$$
\left|X-\mathbb{E}\left[\mathscr{X}_{m}\right]\right| \leq \mu_{\infty} m^{-\gamma}, \quad m \in \mathbb{N}
$$

and

$$
\mathbb{E}\left[\mathscr{X}_{m_{l}}-\mathscr{X}_{m_{l-1}}\right]^{2} \leq \mathscr{V}_{\infty} m_{l}^{-\beta}, \quad l=1, \ldots, L .
$$

These assumptions immediately imply

$$
\left|\mathbb{E}\left[X_{\mathbf{n}, \mathbf{m}}\right]-X\right| \leq \mu_{\infty} m_{L}^{-\gamma}
$$

and

$$
\operatorname{Var}\left[X_{\mathbf{n}, \mathbf{m}}\right] \leq \frac{\sigma_{\infty}^{2}}{n_{0}}+\sum_{l=1}^{L} \frac{\mathscr{V}_{\infty}}{n_{l} m_{l}^{\beta}} .
$$

Note that in the case of the multilevel policy iteration algorithm we have (under assumptions of Theorem 3 ) $\gamma=(1+\alpha) / 2$ and $\beta=\alpha / 2$, where typically $\alpha \geq 1$. For the multilevel dual algorithm it obviously holds (under premises of Theorem 4) $\gamma=(\xi+1) / 2$ and $\beta=1$.

Theorem 5 Let us assume that $0<\beta<1, \gamma \geq \frac{1}{2}$ and $m_{l}=m_{0} \kappa^{l}$ for some fixed $\kappa$ and $m_{0} \in \mathbb{N}$. Fix some $0<\varepsilon<1$. Let $L=L(\varepsilon)$ be the integer part of

$$
\frac{1}{\gamma \ln \kappa} \ln \left[\frac{\sqrt{2} \mu_{\infty}}{m_{0}^{\gamma} \varepsilon}\right],
$$

and

$$
\begin{aligned}
& n_{l}=n_{0} \kappa^{-l(1+\beta) / 2} \quad \text { with } \\
& n_{0}=n_{0}(\varepsilon)=\frac{2 \sigma_{\infty}^{2}}{\varepsilon^{2}}+\frac{2 \mathscr{V}_{\infty}}{\varepsilon^{2} m_{0}^{\beta}} \frac{\kappa^{L(1-\beta) / 2}-1}{\kappa^{(1-\beta) / 2}-1} \kappa^{(1-\beta) / 2} .
\end{aligned}
$$

Then the number of numerical operations needed to achieve the accuracy $\varepsilon$, i.e., to get $\sqrt{\mathbb{E}\left[\left(X-X_{\mathbf{n}, \mathbf{m}}\right)^{2}\right]}<\varepsilon$ is given, up to a constant, by

$$
\begin{aligned}
\mathscr{C}_{\mathrm{ML}}^{\mathbf{n}, \mathbf{m}}(\varepsilon) & =n_{0} m_{0}+\sum_{l=1}^{L} n_{l}\left(m_{l}+m_{l-1}\right) \\
& =\frac{2 \mathscr{V}_{\infty} \kappa^{1-\beta} m_{0}^{1-\beta}\left(1+\kappa^{-1}\right)}{\varepsilon^{2}} \\
& \times\left(\frac{\left(\sqrt{2} \mu_{\infty} / m_{0}^{\gamma} \varepsilon\right)^{(1-\beta) / 2 \gamma}-1}{\kappa^{(1-\beta) / 2}-1}+\frac{\sigma_{\infty}^{2} m_{0}^{\beta}}{\mathscr{V}_{\infty} \kappa^{(1-\beta) / 2}}\right) \\
& \times\left(\frac{\left(\sqrt{2} \mu_{\infty} / m_{0}^{\gamma} \varepsilon\right)^{(1-\beta) / 2 \gamma}-1}{\kappa^{(1-\beta) / 2}-1}+\frac{\kappa^{-(1-\beta) / 2}}{\left(1+\kappa^{-1}\right)}\right) \\
& =O\left(\varepsilon^{-2-\frac{1-\beta}{\gamma}}\right), \quad \varepsilon \searrow 0 .
\end{aligned}
$$




\section{Belomestny, Ladkau, and Schoenmakers}

Corollary 6 By letting $\beta \nearrow 1$ we derive from Theorem 5 that under the choice

$$
\begin{aligned}
& n_{l}=n_{0} \kappa^{-l} \quad \text { with } \\
& n_{0}=n_{0}(\varepsilon)=\frac{2 \sigma_{\infty}^{2}}{\varepsilon^{2}}+\frac{2 \mathscr{V}_{\infty}}{\varepsilon^{2} m_{0}} L,
\end{aligned}
$$

for $\beta=1$ it holds

$$
\mathscr{C}_{\mathrm{ML}}^{\mathbf{n}, \mathbf{m}}(\varepsilon)=O\left(\varepsilon^{-2} \ln ^{2} \varepsilon\right), \quad \varepsilon \searrow 0 .
$$

(cf. the situation in Belomestny and Schoenmakers 2011).

Let $\mathscr{C}_{\text {stan }}^{N, M}(\varepsilon)$ stand for the numerical complexity of the standard Monte Carlo estimate of $X$ based on $M$ inner and $N$ outer paths.

Corollary 7 It holds for $\beta<1$,

$$
\frac{\mathscr{C}_{\mathrm{ML}}^{\mathbf{n}, \mathbf{m}}(\varepsilon)}{\mathscr{C}_{\mathrm{stan}}^{N, M}(\varepsilon)}=O\left(\varepsilon^{\beta / \gamma}\right), \quad \varepsilon \searrow 0
$$

and for $\beta=1$,

$$
\frac{\mathscr{C}_{\mathrm{ML}}^{\mathbf{n}, \mathbf{m}}(\varepsilon)}{\mathscr{C}_{\mathrm{stan}}^{N, M}(\varepsilon)}=O\left(\varepsilon^{1 / \gamma} \ln ^{2} \varepsilon\right), \quad \varepsilon \searrow 0 .
$$

\section{ACKNOWLEDGMENTS}

This research was partially supported by the DFG Research Center MATHEON 'Mathematics for Key Technologies' in Berlin, the DFG project SPP 1324 "Mathematical methods for extracting quantifiable information from complex systems", and by Laboratory for Structural Methods of Data Analysis in Predictive Modeling, MIPT, RF government grant, ag. 11.G34.31.0073.

\section{REFERENCES}

Andersen, L., and M. Broadie. 2004. "A Primal-Dual Simulation Algorithm for Pricing Multi-Dimensional American Options.”. Management Science 50 (9): 1222-1234.

Bally, V., and G. Pages. 2003. "A quantization algorithm for solving multidimensional discrete optimal stopping problem.". Bernoulli 9 (6): 1003-1049.

Belomestny, D., C. Bender, and J. Schoenmakers. 2009. "True upper bounds for Bermudan products via non-nested Monte Carlo". Math. Finance 19 (1): 53-71.

Belomestny, D., M. Ladkau, and J. Schoenmakers. 2012. "Simulation based policy iteration for American style derivatives - a multilevel approach.”. WIAS preprint 1721.

Belomestny, D., and J. Schoenmakers. 2011. "Multilevel dual approach for pricing American style derivatives.". WIAS preprint 1647, Finance Stoch. under revision.

Bender, C., A. Kolodko, and J. Schoenmakers. 2008. "Enhanced policy iteration for American options via scenario selection". Quant. Finance 8 (2): 135-146.

Broadie, M., and P. Glasserman. 2004. "A stochastic mesh method for pricing high-dimensional American options.”. J. Comp. Finance 7 (4): 35-72.

Carriere, J. 1996. "Valuation of the early-exercise price for options using simulations and nonparametric regression.”. Insur. Math. Econ. 19 (1): 19-30.

Davis, M.H.A. and Karatzas, I. 1994. "A deterministic approach to optimal stopping.”. Kelly, F. P. (ed.), Probability, statistics and optimisation. A tribute to Peter Whittle. Chichester: Wiley. Wiley Series in Probability and Mathematical Statistics. Probability and Mathematical Statistics. 455-466. 
Giles, M. B. 2008. "Multilevel Monte Carlo path simulation”. Oper. Res. 56 (3): 607-617.

Glasserman, P. 2004. Monte Carlo methods in financial engineering, Volume 53 of Applications of Mathematics (New York). New York: Springer-Verlag. Stochastic Modelling and Applied Probability.

Haugh, M., and L. Kogan. 2004. "Pricing American options: A duality approach.". Oper. Res. 52 (2): $258-270$.

Howard, R. A. 1960. Dynamic programming and Markov processes. The Technology Press of M.I.T., Cambridge, Mass.

Kolodko, A., and J. Schoenmakers. 2006. "Iterative construction of the optimal Bermudan stopping time.". Finance Stoch. 10 (1): 27-49.

Longstaff, F., and E. Schwartz. 2001. "Valuing American options by simulation: a simple least-squares approach.". Review of Financial Studies 14 (1): 113-147.

Rogers, L. 2002. "Monte Carlo valuation of American options.". Math. Finance 12 (3): 271-286.

Tsitsiklis, J., and B. Van Roy. 2001. "Regression methods for pricing complex American style options.". IEEE Trans. Neural. Net. 12 (14): 694-703.

\section{AUTHOR BIOGRAPHIES}

DENIS BELOMESTNY is a full Professor of Stochastics and Statistics at the Duisburg-Essen University. Prior to joining the university he was a senior researcher at the Weierstrass Institute of Applied Mathematics in Berlin. He holds a Ph.D. from Moscow Lomonosov State University. His email address is denis.belomestny@uni-due.de and his web page is https://www.uni-due.de/ hm0124.

MARCEL LADKAU received a Diploma degree in Mathematics from the Humboldt University Berlin and is currently a Ph.D. student in the applied mathematical finance group of J.S. His email address is Marcel.Ladkau@wias-berlin.de and his web page is https://www.wias-berlin.de/ ladkau.

JOHN SCHOENMAKERS is deputy head of the research group Stochastic Algorithms and Nonparametric Statistics at the Weierstrass Institute Berlin and director of the financial mathematics research in this group. He holds a Ph.D. from Delft University and Habilitation from Humboldt University Berlin, where he is currently a visiting professor. His email address is John.Schoenmakers@wias-berlin.de and his web page is https://www.wias-berlin.de/people/schoenma. 